PESQUIMAT, Revista de la F.C.M. de la

Universidad Nacional Mayor de San Marcos

Vol. XIV N², pp. 17-24, Lima - Perú, Agosto 2011

\title{
ESPACIOS CON PESO
}

Nancy Moya*

Resumen: En este artículo, presentamos un estudio analítico de una clase de pesos, damos algunas propiedades y formulamos algunas estimaciones de estos. Definimos los correspondientes espacios de Sobolev con pesos, tratamos de establecer algunas relaciones entre los espacios de Sobolev con peso y sin peso. Construímos las inclusiones de Sobolev con peso entre los espacios de Sobolev con peso.

Palabras clave: Funciones Peso, Espacios de Sobolev con peso. Inclusiones de Sobolev con peso.

\section{WEIGHTED SPACES}

\begin{abstract}
In this paper, we present an analytical study of a class of weights, We formulate some properties and some estimates of these. From finimos corresponding Sobolev spaces with weights, try to establish some relations between Sobolev spaces with weight and weightless. We built Sobolev inclusions weighing between Sobolev spaces with weight.
\end{abstract}

Key words: Weight function, Weighted Sobolev spaces. Weighted Sobolev Embeddings.

\section{Introducción}

En este artículo hacemos un estudio analítico de una clase de pesos y damos algunas propiedades de los mismos. Presentamos ejemplos importantes de pesos e introducimos una amplia clase de pesos $R_{\rho_{1}, \rho_{2}, \ldots \rho_{k}}$ y formulamos algunas estimaciones de estos.

En la Sección $\S$ ??, definimos la clase de funciones peso $R_{\rho_{1}, \rho_{2}, \ldots \rho_{k}}$, para algunas constantes $\rho_{1}, \rho_{2}, \ldots \rho_{k}$ positivas, los cuales son definidos como pesos que pertenecen a $C^{k}\left(\mathbb{R}^{N},(0, \infty)\right)$ y para $x \in \mathbb{R}^{N},|\alpha|=r$, con $1 \leq r \leq k$, satisfaciendo la siguiente desigualdad

$$
\left|D^{\alpha} \rho(x)\right| \leq \rho_{r} \rho(x), x \in \mathbb{R}^{N} .
$$

Probamos que si $\rho$ está en la clase $R_{\rho_{1}, \rho_{2}}$, entonces su reescalamiento definido por $\rho_{\epsilon}(x)=\rho(\epsilon x)$, tambien está en la clase $R_{\epsilon \rho_{1}, \epsilon^{2}, \rho_{2}}$. Asimismo, los pesos de esta clase verifican estimaciones del tipo $\rho(x) \leq \rho(x-y) e^{\sqrt{N} \rho_{1}|y|}, \quad x, y \in \mathbb{R}^{N}$. En particular, si $x=y$, tenemos que $\rho(x) \leq e^{C|x|} \rho(0)$, donde $C=\sqrt{N} \rho_{1}$. Esto significa que los pesos de la clase $R_{\rho_{1}, \rho_{2}}$ tienen a lo más un crecimiento exponencial en el infinito.

En la Sección § ?? definimos los correspondientes espacios de Sobolev con esos pesos, y analizamos las propiedades importantes de estos espacios. Tratamos de establecer algunas relaciones entre los espacios de Sobolev con peso y sin peso.

En la Sección § ?? Con las herramientas de la Sección anterior construimos las inclusiones de Sobolev con peso entre los espacios $W_{\rho}^{k, p}\left(\mathbb{R}^{N}\right)$ y $L_{\rho^{q}}^{q}\left(\mathbb{R}^{N}\right)$. Finalizamos esta sección analizando cuando es posible establecer las inclusiones de Sobolev con peso de manera análoga a las inclusiones de Sobolev sin peso

$$
W_{\rho}^{k, p}\left(\mathbb{R}^{N}\right) \hookrightarrow L_{\rho}^{q}\left(\mathbb{R}^{N}\right)
$$

y cuando no es posible, para ello estudiamos el comportamiento de los pesos.

${ }^{*}$ UNMSMS, Facultad de Ciencias Matemáticas, e-mail:nanesp@yahoo.com 


\section{Funciones peso}

Una función $\rho: \mathbb{R}^{N} \rightarrow(0, \infty)$ continua y estrictamente positiva sera denominada función peso.

Requeriremos propiedades adicionales de $\rho$, como es la regularidad.

Definición 2.1. Diremos que una función peso $\rho: \mathbb{R}^{N} \rightarrow(0, \infty)$ es una función de la clase $R_{\rho_{1}, \ldots, \rho_{k}}$, con $k \in \mathbb{N}$ si:

(i) $\rho \in C^{(k)}\left(\mathbb{R}^{N}\right)$

(ii) $\left|D^{\alpha} \rho(x)\right| \leq \rho_{r} \rho(x)$, para $x \in \mathbb{R}^{N},|\alpha|=r$, con $1 \leq r \leq k$, y para ciertas constantes positivas $\rho_{r}$.

En particular, se dirá que $\rho$ está en la clase $R_{\infty}$ si se verifica la Definición ?? para todo $k \in \mathbb{N}$.

Lema 2.1. Sea $\rho$ un peso de la clase $R_{\rho_{1}, \rho_{2}}$. Entonces se tiene:

(i) $\rho_{\epsilon}(x):=\rho(\epsilon x)$ es un peso de la clase $R_{\epsilon \rho_{1}, \epsilon^{2} \rho_{2}}$.

(ii) $\rho(x) \leq \rho(x-y) e^{\sqrt{N} \rho_{1}|y|}, x, y \in \mathbb{R}^{N}$.

Algunos ejemplos importantes ver en [?].

Además estos pesos verifican las siguientes desigualdades, que son muy útiles en el estudio de las propiedades de regularización de la solución de la ecuación del calor, que formulamos en el siguiente lema

Lema 2.2. (i) Para todo $\rho \in R_{\rho_{1}, \rho_{2}}, \frac{\rho(x)}{\rho(y)} \leq e^{\sqrt{N} \rho_{1}|x-y|}$.

(ii) Si $\rho(x)=\left(1+|x|^{2}\right)^{\gamma}$, con $\gamma \in \mathbb{R}$ tenemos que

$$
\frac{\rho(x)}{\rho(y)} \leq C(\gamma)\left(1+|x-y|^{2}\right)^{|\gamma|}
$$

y la siguiente condición de integrabilidad en $\mathbb{R}^{N}$

$$
\int_{\mathbb{R}^{N}}\left(1+|x|^{2}\right)^{\gamma} d x<\infty \text { si y sólo si } \gamma<\frac{-N}{2} .
$$

(iii) Si $\rho \in C^{2}\left(\mathbb{R}^{N}\right)$, tal que $\rho(x)=e^{\gamma|x|}$ si $|x| \geq 1$, con $\gamma \in \mathbb{R}$, entonces satisface,

$$
\frac{\rho(x)}{\rho(y)} \leq C e^{|\gamma||x-y|} \text { para cada } x, y \in \mathbb{R}^{N}
$$

y es integrable si $\gamma<0$.

Para la demostración ver [?].

Ahora, presentamos el siguiente lema, que será útil en la siguiente sección

Lema 2.3. Si $\rho \in R=R_{\rho_{1}, \rho_{2}, \ldots, \rho_{n}}$ entonces

$$
\left|D^{\alpha} \rho^{w}(x)\right| \leq C \rho^{w}(x), \text { para cada }|\alpha| \leq n, w \in \mathbb{R} .
$$

donde $C=C\left(n, w, \rho_{1}, \rho_{2}, \ldots, \rho_{n}\right)$.

En particular, $\forall w \in \mathbb{R}, \rho^{w} \in R_{\hat{\rho}_{1}, \ldots, \hat{\rho}_{n}}$ con $\hat{\rho}_{1}=\ldots=\hat{\rho}_{n}=C$, y si $w \in(0,1)$ entonces $\hat{\rho}_{i}$ se puede tomar independiente de $w$.

\section{Espacios con peso.}

Sea $\rho: \mathbb{R}^{N} \rightarrow(0, \infty)$ un peso. Definimos los espacios de Lebesgue con peso de la siguiente manera,

Definición 3.1. El espacio $L^{p}\left(\mathbb{R}^{N}\right)$ con peso $\rho$ se define como

$$
L_{\rho}^{p}\left(\mathbb{R}^{N}\right):=\left\{u \in L_{l o c}^{p}\left(\mathbb{R}^{N}\right): \int_{\mathbb{R}^{N}}|u(x)|^{p} \rho(x) d x<\infty\right\}, 1 \leq p<\infty
$$


con norma

$$
\|u\|_{L_{\rho}^{p}\left(\mathbb{R}^{N}\right)}=\left(\int_{\mathbb{R}^{N}}|u(x)|^{p} \rho(x) d x\right)^{\frac{1}{p}}
$$

Cuando $p=\infty$ definimos el espacio

$$
L_{\rho}^{\infty}\left(\mathbb{R}^{N}\right):=\left\{u \in L_{l o c}^{\infty}\left(\mathbb{R}^{N}\right): \sup _{x \in \mathbb{R}^{N}}|u(x)| \rho(x)<\infty\right\}
$$

con norma

$$
\|u\|_{L_{\rho}^{\infty}\left(\mathbb{R}^{N}\right)}:=\sup _{x \in \mathbb{R}^{N}}|u(x)| \rho(x) .
$$

Análogamente definimos los espacios de Sobolev con peso $W_{\rho}^{k, p}\left(\mathbb{R}^{N}\right)$.

Definición 3.2. Para $k \in \mathbb{N}, 1 \leq p \leq \infty$ denotamos por $W_{\text {loc }}^{k, p}\left(\mathbb{R}^{N}\right)$ el espacio de las $\phi \in L_{\text {loc }}^{p}\left(\mathbb{R}^{N}\right)$ que tienen derivadas distribucionales $D^{\alpha} \phi \in L_{\text {loc }}^{p}\left(\mathbb{R}^{N}\right)$ para todo $|\alpha| \leq k$.

También definimos, $W_{\rho}^{k, p}\left(\mathbb{R}^{N}\right)$ como el espacio de Banach que consiste de todas las $\phi \in W_{\text {loc }}^{k, p}\left(\mathbb{R}^{N}\right)$ tal. que la norma

$$
\|\phi\|_{W_{\rho}^{k, p}\left(\mathbb{R}^{N}\right)}:=\sum_{|\alpha| \leq k}\left\|D^{\alpha} \phi\right\|_{L_{\rho}^{p}\left(\mathbb{R}^{N}\right)}<\infty
$$

En particular cuando $k=1$ y $p=2$ tenemos los espacios,

$$
H_{\rho}^{1}\left(\mathbb{R}^{N}\right)=\left\{u \in L_{\rho}^{2}\left(\mathbb{R}^{N}\right), \nabla u \in\left[L_{\rho}^{2}\left(\mathbb{R}^{N}\right)\right]^{N}\right\}
$$

dotado de la norma

$$
\|u\|_{H_{\rho}^{1}\left(\mathbb{R}^{N}\right)}^{2}=\|u\|_{L_{\rho}^{2}\left(\mathbb{R}^{N}\right)}^{2}+\|\nabla u\|_{\left[L_{\rho}^{2}\left(\mathbb{R}^{N}\right)\right]^{N}}^{2}
$$

Observación 3.1. Si el peso es tal que, $0<m \leq \rho(x) \leq M$, para cada $x \in \mathbb{R}^{N}$, entonces los espacios $L_{\rho}^{p}\left(\mathbb{R}^{N}\right)$ coinciden con los espacios $L^{p}\left(\mathbb{R}^{N}\right)$, con norma equivalente y de la misma manera se tiene que los espacios de Sobolev con peso $W_{\rho}^{k, p}\left(\mathbb{R}^{N}\right)$ coinciden con los espacios de Sobolev standard sin peso $W^{k, p}\left(\mathbb{R}^{N}\right)$.

Por tanto, los casos naturales a considerar son: a) cuando los pesos tienden al infinito en el infinito y b) cuando los pesos tienden a cero en el infinito. Dentro de este caso tenemos el subcaso formado por los pesos integrables. Una propiedad importante de estos últimos es que contienen a las constantes; es más, que $L^{\infty}\left(\mathbb{R}^{N}\right)$ está contenido en $L_{\rho}^{p}\left(\mathbb{R}^{N}\right)$.

Veamos ahora algunas relaciones entre los espacios de Sobolev con peso y sin peso.

Lema 3.1. (i) Si $0<m \leq \rho(x)$ entonces $W_{\rho}^{k, p}\left(\mathbb{R}^{N}\right) \subset W^{k, p}\left(\mathbb{R}^{N}\right)$, y si lím $\lim _{|x| \rightarrow+\infty} \rho(x)=+\infty$ entonces $W_{\rho}^{k, p}\left(\mathbb{R}^{N}\right) \subsetneq W^{k, p}\left(\mathbb{R}^{N}\right), k \in \mathbb{N} \cup\{0\}, 1 \leq p \leq \infty$.

(ii) Si $0<\rho(x) \leq M$ entonces $W^{k, p}\left(\mathbb{R}^{N}\right) \subset W_{\rho}^{k, p}\left(\mathbb{R}^{N}\right)$, y si lím $\lim _{|x|+\infty} \rho(x)=0$ entonces $W^{k, p}\left(\mathbb{R}^{N}\right) \subsetneq W_{\rho}^{k, p}\left(\mathbb{R}^{N}\right), \quad k \in \mathbb{N} \cup\{0\}, 1 \leq p \leq \infty$.

(iii) Si $0<m \leq \rho(x) \leq M$, para cada $x \in \mathbb{R}^{N}, W_{\rho}^{k, p}\left(\mathbb{R}^{N}\right)=W^{k, p}\left(\mathbb{R}^{N}\right)$, para $k \in \mathbb{N} \cup\{0\}$, con normas equivalentes.

(iv) Si $\rho \in L^{1}\left(\mathbb{R}^{N}\right)$ entonces $L^{\infty}\left(\mathbb{R}^{N}\right) \subset L_{\rho}^{p}\left(\mathbb{R}^{N}\right)$, para cada $1 \leq p<\infty$ y si $p \geq q$ entonces $L_{\rho}^{p}\left(\mathbb{R}^{N}\right) \hookrightarrow L_{\rho}^{q}\left(\mathbb{R}^{N}\right)$.

(v) En cualquier caso $L^{p}\left(\mathbb{R}^{N}\right) \cap L_{\rho}^{p}\left(\mathbb{R}^{N}\right)$ es denso en $L_{\rho}^{p}\left(\mathbb{R}^{N}\right)$, para $1 \leq p<\infty$.

Establecemos ahora un isomorfismo isométrico entre espacios de Sobolev con peso y espacios de Sobolev sin peso, el cual nos va a permitir trasladar consideraciones y propiedades de éstos últimos a los primeros. 
Proposición 3.1. Sea $1 \leq p<\infty, \rho \in R=R_{\rho_{1}, \ldots, \rho_{k}}$ con $k \in \mathbb{N} \cup\{0\}$. La aplicación

$$
\begin{array}{ccc}
J: W_{\rho}^{k, p}\left(\mathbb{R}^{N}\right) & \longrightarrow & W^{k, p}\left(\mathbb{R}^{N}\right) \\
u & \longrightarrow & J(u)
\end{array}:=u \rho^{1 / p}
$$

es un isomorfismo. Además si $k=0, J$ es una isometría.

En el caso que $p=\infty, J$ se define como

$$
\begin{array}{ccc}
J: W_{\rho}^{k, \infty}\left(\mathbb{R}^{N}\right) & \longrightarrow & W^{k, \infty}\left(\mathbb{R}^{N}\right) \\
u & \longrightarrow & J(u)
\end{array}:=u \rho
$$

que es un isomorfismo y si $k=0 \mathrm{~J}$ es una isometría.

Observación 3.2. Obsérvese que mediante la aplicación $J$ podemos definir normas equivalentes en los espacios $W_{\rho}^{k, p}\left(\mathbb{R}^{N}\right)$ con $k \in \mathbb{N} \cup\{0\}$ de la siguiente forma

$$
\|u\|_{W_{\rho}^{k, p}\left(\mathbb{R}^{N}\right)}:=\left\|u \rho^{\frac{1}{p}}\right\|_{W^{k, p}\left(\mathbb{R}^{N}\right)} .
$$

Para las aplicaciones a las ecuaciones en derivadas parciales es útil considerar los espacios intermedios a los definidos arriba. Para ello haremos uso de la noción de Interpolación, que resumimos de la siguiente manera. Sean $A_{0}, A_{1}$ dos espacios de Banach. Para cada $0<\theta<1$ se le asocia el espacio intermedio o de interpolación entre $A_{0}$ y $A_{1}$ denotado por $\left[A_{0}, A_{1}\right]_{\theta}$, que verifica las siguientes propiedades:

(i) Si $A_{0} \subset A_{1}$ entonces para $0<\theta<1, A_{0} \subset\left[A_{0}, A_{1}\right]_{\theta} \subset A_{1}$.

(ii) Para cada $0<\theta<1$, existe un número positivo $c_{\theta}$, tal que para todo $a \in A_{0} \cap A_{1}$ se cumple la. siguiente estimación

$$
\|a\|_{\left[A_{0}, A_{1}\right]_{\theta}} \leq c_{\theta}\|a\|_{A_{0}}^{\theta}\|a\|_{A_{1}}^{1-\theta}
$$

denominada también desigualdad de interpolación.

(iii) Sean los siguientes espacios de interpolación $A_{\theta}:=\left[A_{0}, A_{1}\right]_{\theta}$ y $B_{\theta}:=\left[B_{0}, B_{1}\right]_{\theta}$. Si $T: A_{0} \rightarrow B_{0}$ y $T: A_{1} \rightarrow B_{1}$ son aplicaciones lineales y continuas entonces $T:\left[A_{0}, A_{1}\right]_{\theta} \rightarrow\left[B_{0}, B_{1}\right]_{\theta}$ con $0<\theta<1$, también es una aplicación lineal y continua. En particular, si son isomorfismos, entonces $T:\left[A_{0}, A_{1}\right]_{\theta} \rightarrow\left[B_{0}, B_{1}\right]_{\theta}$ con $0<\theta<1$, también es un isomorfismo. Para más detalles ver [?].

La siguiente definición es válida para cualquier tipo de interpolación. Nosotros en adelante utilizamos la interpolación compleja, por que es la que permite caracterizar los espacios de potencias fraccionarias, como se verá mas adelante. Ver [?].

Definición 3.3. Para $1 \leq p \leq \infty, k \in \mathbb{N} \cup\{0\}$ y $s \in(k, k+1)$ definimos $\theta \in(0,1)$ tal que $s=(1-\theta) k+\theta(k+1)$, esto es $\theta=s-k$. Entonces definimos los espacios intermedios de la siguiente manera

$$
W^{s, p}\left(\mathbb{R}^{N}\right):=\left[W^{k+1, p}\left(\mathbb{R}^{N}\right), W^{k, p}\left(\mathbb{R}^{N}\right)\right]_{\theta},
$$

$y$

$$
W_{\rho}^{s, p}\left(\mathbb{R}^{N}\right):=\left[W_{\rho}^{k+1, p}\left(\mathbb{R}^{N}\right), W_{\rho}^{k, p}\left(\mathbb{R}^{N}\right)\right]_{\theta} .
$$

El siguiente lema es una extensión de la Proposición ?? a espacios intermedios

Lema 3.2. Sea $1 \leq p<+\infty, \rho \in R_{\rho_{1}, \rho_{2}, \ldots . . \rho_{k}}, s \notin \mathbb{N}$ tal que $s \in(r, r+1)$ con $r \in \mathbb{N}$ y $0 \leq r \leq k-1$ entonces

$$
\begin{array}{ccc}
J: W_{\rho}^{s, p}\left(\mathbb{R}^{N}\right) & \longrightarrow & W^{s, p}\left(\mathbb{R}^{N}\right) \\
u & \longrightarrow & J(u)
\end{array}=u \rho^{1 / p}
$$

es un isomorfismo.

Si $p=\infty$, entonces

$$
\begin{array}{clc}
J: W_{\rho}^{s, \infty}\left(\mathbb{R}^{N}\right) & \longrightarrow & W^{s, \infty}\left(\mathbb{R}^{N}\right) \\
u & \longrightarrow & J(u)
\end{array}=u \rho
$$

es un isomorfismo. 


\section{Inclusiones de Sobolev para espacios con peso.}

A continuación establecemos las inclusiones de Sobolev con peso entre los espacios $W_{\rho}^{k, p}\left(\mathbb{R}^{N}\right)$ y $L_{\rho^{p}}^{q}\left(\mathbb{R}^{N}\right)$ con la funcion peso $\rho$ perteneciente a la clase $R=R_{\rho_{1}, \rho_{2}, \ldots, \rho_{k}}$. Estos resultados se utilizan para controlar los teoremas de existencia; su buena definición es consecuencia del isomorfismo $J$, que permite bajar al espacio de Sobolev $W^{k, p}\left(\mathbb{R}^{N}\right)$ y alli hacer uso de las inclusiones de Sobolev sin peso $W^{k, p} \hookrightarrow L^{q}\left(\mathbb{R}^{N}\right)$, luego usamos el isomorfismo isométrico $J$ para volver a los espacios con peso. Lo enunciamos de manera precisa en el siguiente lema.

Lema 4.1. Si $1 \leq p<\infty$ tenemos,

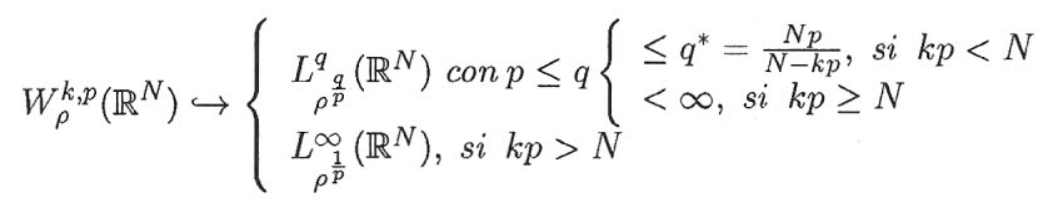

Demostración. La demostración se sintetiza en el siguiente diagrama conmutativo, siempre que $q$ sea finito.

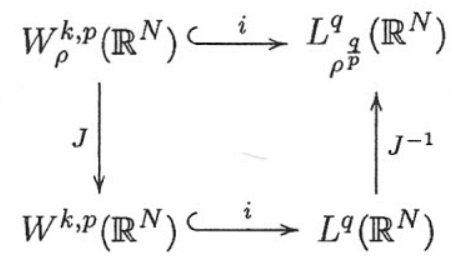

y si $q=\infty$,

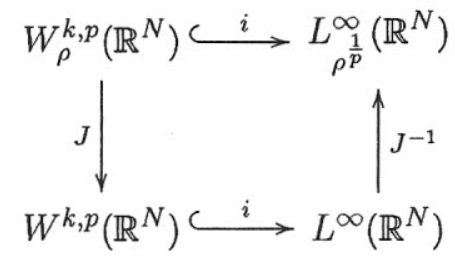

1) Sabemos que $u \in W_{\rho}^{k, p}\left(\mathbb{R}^{N}\right)$ si y sólo si $J(u)=u \rho^{\frac{1}{p}} \in W^{k, p}\left(\mathbb{R}^{N}\right)$ (por la Proposición ??).

2) Por las desigualdades habituales de Sobolev $W^{k, p}\left(\mathbb{R}^{N}\right) \hookrightarrow L^{q}\left(\mathbb{R}^{N}\right), p \leq q \leq q^{*}$ con $q^{*}=q^{*}(p)$, como en (??). Por tanto tenemos que $J(u)=u \rho^{\frac{1}{p}} \in L^{q}\left(\mathbb{R}^{N}\right)$.

3) Si $q<\infty$ entonces $\int_{\mathbb{R}^{N}}|u(x)|^{q} \rho^{q}(x) d x<\infty$ es decir $u \in L_{\rho^{q} p}^{q}\left(\mathbb{R}^{N}\right)$.

4) Si $q=\infty$ entonces $u \rho^{\frac{1}{p}} \in L^{\infty}\left(\mathbb{R}^{N}\right)$ si y sólo si $u \in L_{\rho^{\frac{1}{p}}}^{\infty}\left(\mathbb{R}^{N}\right)$.

En particular

Lema 4.2.

$$
H_{\rho}^{1}\left(\mathbb{R}^{N}\right) \hookrightarrow\left\{\begin{array}{l}
L_{\rho^{\frac{1}{2}}}^{\infty}(\mathbb{R}), \text { si } N=1 \\
L_{\rho^{\frac{p}{2}}}^{p}\left(\mathbb{R}^{N}\right), \text { con } 2 \leq p\left\{\begin{array}{l}
<\infty, \text { si } N=1,2 \\
\leq \frac{2 N}{N-2}, \text { si } N \geq 3
\end{array}\right.
\end{array}\right.
$$

Vamos a extender aquí las inclusiones para espacios intermedios, sean $s_{1}, s_{2} \notin \mathbb{N}$,

Lema 4.3. Sea $\rho \in R_{\rho_{1}, \ldots, \rho_{k}}$, si $0 \leq s_{2} \leq s_{1}<k, 1<p_{1} \leq p_{2}<\infty$ y $s_{1}-\frac{N}{p_{1}} \geq s_{2}-\frac{N}{p_{2}}$ entonces tenemos la siguiente inclusión

$$
W_{\rho}^{s_{1}, p_{1}}\left(\mathbb{R}^{N}\right) \subset W_{\rho^{p_{2}}}^{s_{2, p_{2}}^{p_{2}}}\left(\mathbb{R}^{N}\right)
$$

$S i p_{2}=\infty$ bajo las mismas condiciones anteriores tenemos la siguiente inclusión

$$
W_{\rho}^{s_{1}, p_{1}}\left(\mathbb{R}^{N}\right) \subset W_{\rho^{\frac{1}{p_{1}}}}^{s_{2}, \infty}\left(\mathbb{R}^{N}\right)
$$


Demostración. Si $p_{2}<\infty$, consideramos el siguiente diagrama conmutativo

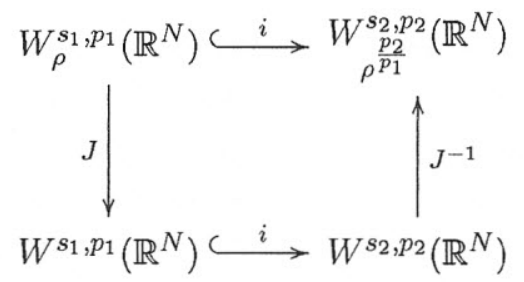

Sea $u \in W_{\rho}^{s_{1}, p_{1}}\left(\mathbb{R}^{N}\right)$ entonces por el isomorfismo $J$ del Lema ?? se tiene que $J(u):=u \rho^{\frac{1}{p_{1}}} \in W^{s_{1}, p_{1}}\left(\mathbb{R}^{N}\right)$ pero como por hipótesis $s_{1}-\frac{N}{p_{1}} \geq s_{2}-\frac{N}{p_{2}}$ tenemos que,

$$
W^{s_{1}, p_{1}}\left(\mathbb{R}^{N}\right) \hookrightarrow W^{s_{2}, p_{2}}\left(\mathbb{R}^{N}\right) .
$$

Entonces $u \rho^{\frac{1}{p_{1}}} \in W^{s_{2}, p_{2}}\left(\mathbb{R}^{N}\right)$, ahora, tenemos que determinar un peso $\varphi$ tal que por el isomorfismo visto en el Lema??,

$$
\begin{array}{ccc}
J: W_{\varphi}^{s_{2}, p_{2}}\left(\mathbb{R}^{N}\right) & \longrightarrow & W^{s_{2}, p_{2}}\left(\mathbb{R}^{N}\right) \\
u & \longrightarrow & J(u)
\end{array}=u \varphi^{1 / p_{2}}
$$

se cumpla que $u \varphi^{1 / p_{2}}=u \rho^{\frac{1}{p_{1}}}$. De aquí $\varphi=\rho^{\frac{p_{2}}{p_{1}}}$ y por tanto obtenemos, $u \in W_{\rho^{\frac{p}{p_{1}}}}^{s_{2}, p_{2}}\left(\mathbb{R}^{N}\right)$.

En el caso que $p_{2}=\infty$ consideramos el siguiente diagrama conmutativo

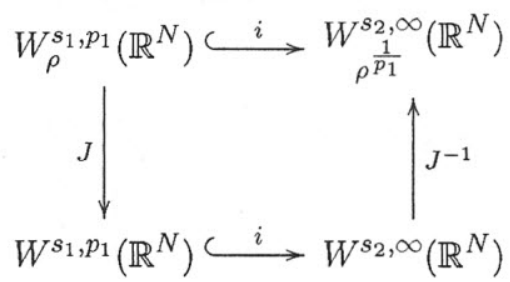

Sea $u \in W_{\rho}^{s_{1}, p_{1}}\left(\mathbb{R}^{N}\right)$, seguimos los mismos pasos como en el caso $p_{2}$ finito, entonces tenemos que $u \rho^{\frac{1}{p_{1}}} \in W^{s_{2}, \infty}\left(\mathbb{R}^{N}\right)$, ahora, tenemos que determinar un peso $\varphi$ tal que por el isomorfismo visto en el Lema?? caso $p_{2}=\infty$, es decir

$$
\begin{array}{clc}
J: W_{\varphi}^{s_{2}, \infty}\left(\mathbb{R}^{N}\right) & \longrightarrow & W^{s_{2}, \infty}\left(\mathbb{R}^{N}\right) \\
u & \longrightarrow & J(u)
\end{array}=u \varphi
$$

se cumpla que $u \varphi=u \rho^{\frac{1}{p_{1}}} ;$ de aquí $\varphi=\rho^{\frac{1}{p_{1}}}$ y por tanto, $u \in \underset{\rho^{\frac{1}{p_{1}}}}{\frac{1}{s_{2}, \infty}}\left(\mathbb{R}^{N}\right)$.

Un caso particular de este lema es cuando $s_{2}=0$ es una extensión del Lema ?? y lo formulamos de la. siguiente manera,

Lema 4.4. Sean $1<p<\infty$, y $s>0$ tenemos que,

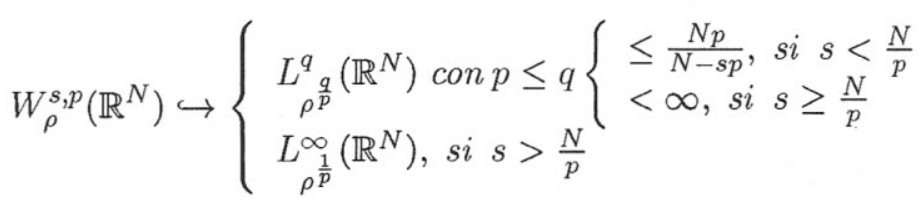

Finalizamos esta sección haciendo una pequeña disgresion sobre pesos y su relación con las inclusiones en los espacios de Sobolev sin peso.

Observación 4.1. Tratamos de encontrar inclusiones de Sobolev con peso análogas a las inclusiones de. Sobolev sin peso, para poder usarlas como instrumento en la resolución de problemas de Cauchy y usar herramientas ya conocidas las técnicas de [?]. 
1.- Para pesos $\rho(x) \rightarrow \infty$, cuando $|x| \rightarrow \infty$ y $\rho(x) \geq 1$, por ejemplo, el peso $\rho(x)=\left(1+|x|^{2}\right)^{\gamma}$ con $\gamma>0$, si $q>p$ se tiene la siguiente inclusión

$$
L_{\rho^{\frac{q}{p}}}^{q}\left(\mathbb{R}^{N}\right) \hookrightarrow L_{\rho}^{q}\left(\mathbb{R}^{N}\right) .
$$

De hecho si $u \in L_{\rho^{\frac{q}{p}}}^{q}\left(\mathbb{R}^{N}\right)$ entonces como $\frac{q}{p}>1$, se verifica la siguiente relación

$$
\int_{\mathbb{R}^{N}}|u(x)|^{q} \rho(x) d x \leq \int_{\mathbb{R}^{N}}|u(x)|^{q} \rho^{\frac{q}{p}}(x) d x<\infty
$$

y por tanto $u \in L_{\rho}^{q}\left(\mathbb{R}^{N}\right)$.

Entonces si estamos en el primer caso del Lema ??, tenemos la cadena

$$
W_{\rho}^{s, p}\left(\mathbb{R}^{N}\right) \hookrightarrow L_{\rho^{p}}^{q}\left(\mathbb{R}^{N}\right) \hookrightarrow L_{\rho}^{q}\left(\mathbb{R}^{N}\right)
$$

con p y q como en el lema. En resumen se tiene la siguiente inclusión que son análogas a las inclusiones de Sobolev sin peso

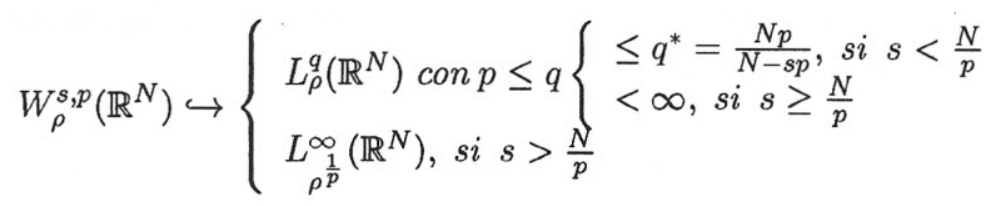

Estas inclusiones son de utilidad a la hora de encontrar soluciones locales de problemas parabólicos en espacios con pesos.

2.- En cambio para pesos $\rho(x) \rightarrow 0$, cuando $|x| \rightarrow \infty$ se pueden construir ejemplos en los que, para $q>p$,

$$
L_{\rho^{\frac{q}{p}}}^{q}\left(\mathbb{R}^{N}\right) \nrightarrow L_{\rho}^{q}\left(\mathbb{R}^{N}\right) .
$$

En efecto, sea $\rho(x)=\left(1+|x|^{2}\right)^{\gamma}$ con $\left.\gamma<0, q\right\rangle p, y u(x)=\left(1+|x|^{2}\right)^{r}$, con $r$ a determinar líneas abajo. Entonces $u \in L_{\rho^{q}}^{q}\left(\mathbb{R}^{N}\right)$ si y sólo si

$$
\int_{\mathbb{R}^{N}}\left(1+|x|^{2}\right)^{q r}\left(1+|x|^{2}\right)^{\gamma \frac{q}{p}} d x=\int_{\mathbb{R}^{N}}\left(1+|x|^{2}\right)^{q r+\frac{\gamma q}{p}} d x<\infty
$$

lo cual se verifica siempre que, $q r+\frac{\gamma q}{p}<-\frac{N}{2}$, por el Lema ??, lo cual equivale a $r<\left(\frac{-N}{2}-\frac{\gamma q}{p}\right) \frac{1}{q}$.

Por otro lado $u \notin L_{\rho}^{q}\left(\mathbb{R}^{N}\right)$ si $r q+\gamma \geq \frac{-N}{2}$, es decir si $r \geq\left(\frac{-N}{2}-\gamma\right) \frac{1}{q}$.

Entonces la prueba acaba si es posible encontrar un $r$ tal que verifica

$$
\left[\frac{-N}{2}-\gamma\right] \frac{1}{q} \leq r<\left[\frac{-N}{2}-\frac{\gamma q}{p}\right] \frac{1}{q}
$$

pero esto último es verdad puesto que se cumple $\left[-\frac{N}{2}-\gamma\right] \frac{1}{q}<\left[\frac{-N}{2}-\frac{\gamma q}{p}\right] \frac{1}{q}$ ya que $\frac{q}{p}>1$.

Por tanto

$$
W_{\rho}^{k, p}\left(\mathbb{R}^{N}\right) \hookrightarrow L_{\rho}^{q}\left(\mathbb{R}^{N}\right) \text { para ningún } q>p,
$$

y no hay inclusiones análogas al caso sin peso. 


\section{Bibliografía}

[1] Brezis, H. (1984). Analisis funcional teoría y aplicaciones. Alianza Editorial.

[2] Henry, D. (1981). Geometric Theory of Semilinear Parabolic Equations, Lecture Notes Math, Vol 840 , Springer, Berlin.

[3] Moya, N. (2009). Revista de Investigación de la Facultad de Ciencia Matemáticas UNMSM. Vol. XII $N^{\circ} 1$.

[4] Triebel, H. (1995). Interpolation Theory Function Spaces Differential Operators. Second edition, Johann Ambrosius Barth Verlag -Heidelberg. Leipzig. 